\title{
ANALYSES OF FACE RECOGNITION RATE USING HOLISTIC METHODS
}

\author{
Romana OANCEA*, Dorel BADEA*, Raluca BÂRSAN** \\ *"Nicolae Bălcescu" Land Forces Academy, Sibiu, Romania \\ **"Lucian Blaga" University, Sibiu, Romania \\ oancea.romana@gmail.com,badea.dorel@yahoo.com
}

\begin{abstract}
The paper focuses on the analysis of holistic facial recognition rates obtained by changing the parameters related to the number of components and the number of images for the same subject in the training set. It has been observed that, regardless of the method used, choosing a small number of samples for the training set does not lead to acceptable results. The recognition rate for using the PCA algorithm is directly influenced by the number of samples and by the size of the feature vector. Thus, if all the features were retained and the number of images in the training set was high, 95\% rates were obtained. Similarly, in the case of the extraction of vectors of variable dimensions with DCT and the use of a neural network for classification, the obtained rates were not satisfactory for a small number of samples in the training set.
\end{abstract}

\section{Keywords: face recognition, PCA, DCT, Neural Network}

\section{Introduction}

Although embedded facial recognition systems are no longer a novelty, research continues in this area and seeks, on the one hand, to improve recognition rates by developing new techniques and methods or combining existing methods, and on the other hand it is desired to extend the areas in which embedded systems are used. If, some time ago, face recognition systems were used in particular for law enforcement and currently in marketing, retail or services, recognition is required in order to know a priori the profile of the buyer and to offer the products he might be interested in. Generally, a facial recognition system is composed of several stages.

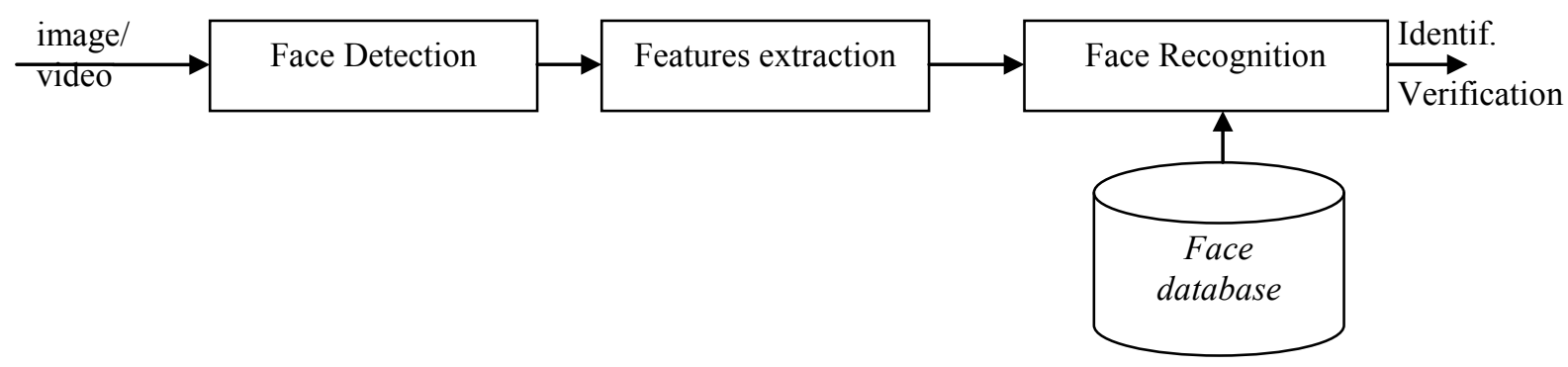

Figure 1. Face recognition system 
Face Detection - involves segmenting the regions corresponding to the faces in the images or sequences, if any. In general, after face segmentation, the lighting and position corrections are applied.

Feature extraction - it is desired to extract the features independent of geometric and photometric variations, and the obtained vector will be used in the recognition stage.

The recognition stage may be of identification or verification [1]. In case of identification, for a new face, the system must be able to determine the identity based on prototypes saved in the database. It is a type 1 problem: $\mathrm{N}$ - for each new face, the database is queried to determine the identity; a "matching" score between the newface and each existing identity in the database is calculated and the identity with the best score is chosen. In the case of verification, the system must accept or deny the identity and in fact it assumes a 1:1 match between a new face and an identity saved in the database.

Face recognition techniques that use an image or video sequence acquired as a source of input in the visible spectrum are classified into three major categories[2, 3]: feature-based, holistic and hybrid.

- Feature - based methods-make the recognition based on the vector formed by the facial features (eyes, nose, mouth) and the geometrical distances between them or, in addition to the specific face features, other measures or characteristics are also considered: edges, lines, curves etc [3].

- Holistic or appearance based methods use a global face recognition. The statistical methods of this representation, such as PCA (Principal Component Analysis), LDA (Linear Discriminant Analysis and ICA (Independent Component Analysis) and variants thereof, are the most commonly used linear classifiers.

- Hybrid methods - combines the holistic methods to reduce the facial features vector and to improve the recognition rate [4].

Most reconfiguration algorithms perform this operation based on images saved first and the number of variants for the same individual is generally low. Except for the situation where an age factor can intervene on an individual, the work is aimed at analyzing the good recognition rates according to the number of samples for each subject. The comparative analysis of the various recognition algorithms was done on the ORL Faces Databases [5]. In the database considered, 10 aspects are captured for 40 individuals. The images, size $112 \times 92$, are purchased under various lighting and face variation conditions, and the facial expressions of the same subject are also varied.

The paper aims at the comparative analysis of the recognition rates for the holistic approach, depending on the number of features and the number of samples for each subject in the training set.

\section{Analysis of recognition rates for holistic approach}

\subsection{ThePCA algorithm}

The PCA algorithm allows the representation of a face as a main component vector. Starting from a set of testing images, the basic vectors are determined from an optimal subspace so that the MSE between the projection of the subspace training images and the original images is minimal. The number of components affects the recognition rate $[6,7]$.

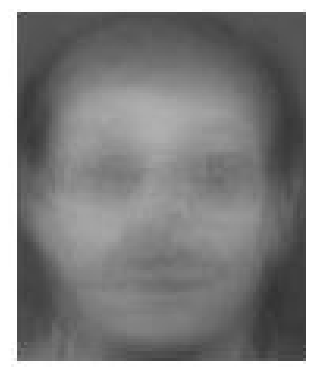

Figure 2. Average face vector 
Table 1 Recognition rates obtained using PCA, variable number of features and variable number of images in the training set

\begin{tabular}{|l|l|l|l|}
\hline $\begin{array}{c}\text { Number of } \\
\text { images in } \\
\text { the } \\
\text { training set }\end{array}$ & $\begin{array}{c}\text { Number } \\
\text { of images } \\
\text { in the } \\
\text { testing } \\
\text { set }\end{array}$ & $\begin{array}{c}\text { Features } \\
\text { number }\end{array}$ & $\begin{array}{c}\text { Recognition } \\
\text { rate }\end{array}$ \\
\hline 360 & 40 & & $95 \%$ \\
\hline 320 & 80 & \multirow{5}{*}{10304} & $95 \%$ \\
\hline 280 & 120 & & $90.83 \%$ \\
\hline 240 & 160 & & $85 \%$ \\
\hline 80 & 320 & & $71.56 \%$ \\
\hline 40 & 360 & & $60.56 \%$ \\
\hline 360 & 40 & & $92.5 \%$ \\
\hline 320 & 80 & & $92.5 \%$ \\
\hline 280 & 120 & \multirow{5}{*}{2576} & $89.17 \%$ \\
\hline 240 & 160 & & $83.13 \%$ \\
\hline 80 & 320 & & $72.19 \%$ \\
\hline 40 & 360 & & $60.28 \%$ \\
\hline
\end{tabular}

It can be noticed that the smaller the number of images in the training set for the same individual, the lower the recognition rate. At the same time, the higher the features vector, the higher the recognition rate. In situations where, for an individual there were one or two states, the likelihood of recognition would be very small. In other words, for identification, this algorithm can not cope with the challenges.

\subsection{LDA algorithm}

The LDA algorithm is a statistical feature selection method, similar to PCA, with the difference that, in classification issues, it determines the hyperplan that separates the classes considered by maximizing the interclasses covariance matrix and minimizing covariance within the same class. LDA is sensitive to occlusions and requires a large number of images in the training set to obtain a correct distribution of dispersions $[8,9,10]$. In addition, if the number of classes is not high enough, it is preferable to apply the PCA method due to better results.
Table 2. Recognition rates obtained using the

$L D A$, variable number of features and the variable number of images in training set

\begin{tabular}{|l|l|l|}
\hline $\begin{array}{c}\text { Number of } \\
\text { images in the } \\
\text { training set }\end{array}$ & $\begin{array}{c}\text { Number of } \\
\text { images in the } \\
\text { testing set }\end{array}$ & $\begin{array}{c}\text { Recognition } \\
\text { rate }\end{array}$ \\
\hline 360 & 40 & 93.57 \\
\hline 320 & 80 & 93.21 \\
\hline 280 & 120 & 94.29 \\
\hline 240 & 160 & 96.07 \\
\hline 80 & 320 & 87.81 \\
\hline 40 & 360 & 63.89 \\
\hline
\end{tabular}

It can be seen that the results of LDA application on a relatively small set of data do not lead to higher results than PCA. If the number of images in the training set is 1 or 2 images per subject, the results are significantly better, but false detection rates are still high. In other words, both the LDA algorithm and the PCA can not be successfully applied if the number of images in the training set is low.

\subsection{Discrete Cosine Transform}

In the recognition process, an important factor is the number of retained features. Applying the cosine transform allows selecting low frequency components containing useful information $[11,12]$.
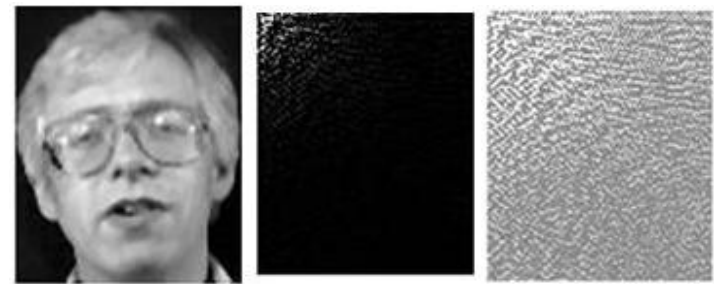

Figure 3. Image of the training set, DCT; histogram equalization for DCT

After selecting the useful components from the transformed cosine, neural networking or other techniques were commonly used in face recognition [13-15].

To analyze the recognition rate based on the number of features retained for an image, following the application of DCT, a neural network with 40 neurons on the hidden layer and the trainscg training function was used. The output layer consists of 40 
neurons corresponding to the 40 subjects. To see how the recognition rate is affected by the number of retained features and the number of images for each class in the training set, a neural network with the input layer was used and tested, depending on the initial data considered.

Table 3. Recognition rate obtained by applying $D C T$, a variable number of features for the input layer, and a variable number of samples

\begin{tabular}{|c|c|c|c|}
\hline & & \multicolumn{2}{|c|}{ for each class trained } \\
\hline $\begin{array}{l}\text { Number of } \\
\text { images in } \\
\text { the } \\
\text { training set }\end{array}$ & $\begin{array}{l}\text { Number } \\
\text { of } \\
\text { images } \\
\text { in the } \\
\text { testing } \\
\text { set }\end{array}$ & $\begin{array}{l}\text { Features } \\
\text { number }\end{array}$ & $\begin{array}{l}\text { Recognition } \\
\text { rate }\end{array}$ \\
\hline 360 & 40 & \multirow{5}{*}{100} & $86.11 \%$ \\
\hline 320 & 80 & & $85.00 \%$ \\
\hline 280 & 120 & & $80.02 \%$ \\
\hline 240 & 160 & & $72.50 \%$ \\
\hline 80 & 320 & & $51.25 \%$ \\
\hline 360 & 40 & \multirow{5}{*}{64} & $87.50 \%$ \\
\hline 320 & 80 & & $88.75 \%$ \\
\hline 280 & 120 & & $84.17 \%$ \\
\hline 240 & 160 & & $85.63 \%$ \\
\hline 80 & 320 & & $55.31 \%$ \\
\hline 360 & 40 & \multirow{5}{*}{32} & $85 \%$ \\
\hline 320 & 80 & & $90.25 \%$ \\
\hline 280 & 120 & & $81.67 \%$ \\
\hline 240 & 160 & & $82.50 \%$ \\
\hline 80 & 320 & & $33.75 \%$ \\
\hline 360 & 40 & \multirow{4}{*}{16} & $87.5 \%$ \\
\hline 320 & 80 & & $86.25 \%$ \\
\hline 280 & 120 & & $76.67 \%$ \\
\hline 240 & 160 & & $71.25 \%$ \\
\hline 360 & 40 & \multirow{4}{*}{8} & $82.50 \%$ \\
\hline 320 & 80 & & $72.50 \%$ \\
\hline 280 & 120 & & $68.33 \%$ \\
\hline 240 & 160 & & $43.12 \%$ \\
\hline
\end{tabular}

Using a neural recognition network, recognition rates of over $90 \%$ can be achieved, and, in addition, for PCA-like performance, the number of features retained in each image is much, much smaller. If for a small number of small samples for the same subject in the training set, the PCA algorithm yields approximately $60 \%$ results, neural network recognition does not reach the $60 \%$ threshold if the number of images is less than 6 for each subject.

\section{Conclusions}

For facial recognition, if the number of images in the training set is low, the recognition rate is also reduced, and holistic algorithms can not be applied successfully. Another parameter that influences the recognition rate is the number of features retained in each image. A large number of features implies a pro-active driving time, but the results, when using holistic methods, are higher. For a small number of features in the training set, but a large number of samples for the same subject, the DCT, together with a neural network, leads to noticeably better results.

As further developments, it is intended that in the face preprocessing stage the initial facial features should be localized, and then the face aligned with the facial features detected. At the same time, an adaptive change of illumination could lead to improved results.

[1] Abate A. F., Nappi M., Riccio D., Sabatin G.., 2D and 3D face recognition: A survey, in Pattern Recognition Letters, vol. 28, no.14, pp. 1885-1906, 2007

[2] Jafri R., Arabnia H.R., A Survey of Face Recognition Techniques, in Journal of Information Processing Systems, Vol.5, No.2, p. 41-68, June 2009

[3] Zhao W., Chellappa R., Phillips P.J., Rosenfeld A., Face recognition: A literature survey, in ACM Comput. Surv. 34 (4), pp. 399-458, 2003

[4] Cho H., Roberts R., Jung B., Choi O., Moon S., An Efficient Hybrid Face Recognition Algorithm Using PCA and GABOR Wavelets, in International Journal of Advanced Robotic Systems, vol. 11 (1), pp.1-8. doi:10.5772/58473, 2014 
[5] http://www.cl.cam.ac.uk/research/dtg/attarchive/facedatabase.html

[6] Kirby M., Sirovich L., Low-dimensional procedure for the characterization of human faces, in journal of the Optical Society of America 4, 3, pp. 519-524, 1987.

[7] Kirby M., Sirovich L., Application of the Karhunen-Loève Procedure for the Characterization of Human Faces, in IEEE Transactions on Pattern Analysis and Machine Intelligence 12, 1, pp. 103-108, 1990

[8] Etemad K., Chellappa R., Discriminant analysis for recognition of human face images. Journal of Optical Society of America 14, 8, pp. 1724-1733, 1997

[9] Martinez A. M., Kak A. C., PCA vs. LDA. IEEE Transactions on PatternAnalysis and Machine Intelligence 23, 2, pp. 228-233, 2001

[10] Zhao W., Chellappaand R., Krishnaswamy A., Discriminant Analysis of Principal Components for Face Recognition.inProceedings of the 3rd IEEE International Conference on Face and Gesture Recognition (FG '98), vol. 2, pp. 336-341, Nara, Japan, 1998.

[11] Manikantan K., Govindarajan V., Face Recognition using Block Based DCT Feature Extraction, Journal of Advanced Computer Science and Technology, 1 (4), 266-283, 2012

[12] Hafed Z. M., Levine M. D., Face Recognition Using the Discrete Cosine Transform, International Journal of Computer Vision, 167-188, 2001.

[13] Nagi J., Ahmed S. K., Nagi F., A MATLAB based Face Recognition System usingImage Processing and Neural Networks, in 4th International Colloquium on Signal Processing and its Applications, March 7-9, Kuala Lumpur, Malaysia, pp. 83- 85, 2008

[14] Aman R. Chadha, Pallavi P. Vaidya, M. Mani Roja, Face Recognition Using Discrete Cosine Transform for Global and Local Features, in Proceedings of the 2011 International Conference on Recent Advancements in Electrical, Electronics and Control Engineering (IConRAEeCE), pp. 1-4, 2011

[15] Zahraddeen Sufyanu, Fatma S. Mohamad, Abdulganiyu A. Yusuf, Mustafa B. Mamat, Enhanced Face Recognition Using Discrete Cosine Transform, in Engineering Letters, 24:1, EL_24_1_08, Advance online publication: 29 February 2016, available at http://www.engineeringletters.com/issues_v24/issue_1/EL_24_1_08.pdf 